MATHEMATICS OF COMPUTATION

Volume 73, Number 248, Pages 1719-1737

S $0025-5718(03) 01621-1$

Article electronically published on December 19, 2003

\title{
ON THE ERROR ESTIMATES FOR THE ROTATIONAL PRESSURE-CORRECTION PROJECTION METHODS
}

\author{
J. L. GUERMOND AND JIE SHEN
}

\begin{abstract}
In this paper we study the rotational form of the pressure-correction method that was proposed by Timmermans, Minev, and Van De Vosse. We show that the rotational form of the algorithm provides better accuracy in terms of the $H^{1}$-norm of the velocity and of the $L^{2}$-norm of the pressure than the standard form.
\end{abstract}

\section{INTRODUCTION}

There are numerous way to discretize the unsteady incompressible Navier-Stokes equations in time. Undoubtedly, the most popular one consists of using projection methods. This class of techniques has been introduced by Chorin and Temam [2, 3, 17]. They are time marching algorithms based on a fractional step technique that may be viewed as a predictor-corrector strategy aiming at uncoupling viscous diffusion and incompressibility effects. The method proposed originally, although simple, is not satisfactory since its convergence rate is irreducibly limited to $O(\delta t)$. This limitation comes from the fact that the method is basically an artificial compressibility technique as shown in [11] and [13. To cure these problems, numerous modifications have been proposed, among which are pressure-correction methods (see [6, 20]) and splitting techniques based on extrapolated pressure boundary conditions (see [10, 9]).

Pressure-correction methods are widely used and have been extensively analyzed. These schemes are composed of two substeps by time step: the pressure is made explicit in the first substep and is corrected in the second one by projecting the provisional velocity onto the space of incompressible vector fields. Rigorous second-order error estimates for the velocity have been proved by E and Liu [4] and Shen [15] in the semi-discrete case and by Guermond [7] and E and Liu [5] in the fully discrete case. We refer also to [16] and 1] for different proofs based on normal mode analysis in the half plane and in a periodic channel, respectively.

It is well known that standard pressure-correction schemes still suffer from the nonphysical pressure boundary condition which induces a numerical boundary layer

Received by the editor February 11, 2002 and, in revised form, March 2, 2003.

2000 Mathematics Subject Classification. Primary 65M12, 35Q30, 76D05.

Key words and phrases. Navier-Stokes equations, projection methods, fractional step methods, incompressibility, finite elements, spectral approximations.

The work of the second author is partially supported by NFS grants DMS-0074283 and DMS0311915. Part of the work was completed while this author was a CNRS "Poste Rouge" visitor at LIMSI. 
and, consequently, degrades the accuracy of the pressure approximation. In 1996, Timmermans, Minev and Van De Vosse [19] proposed a modified version of the pressure-correction scheme, which we shall hereafter refer to as the rotational form of the pressure-correction scheme for reasons we shall specify later, and they showed numerically that it leads to improved pressure approximation. Recently, Brown, Cortez and Minion [1] used normal mode analysis to study the accuracy of this scheme in a periodic channel and showed that the pressure approximation in this particular case is second-order accurate. However, whether the rotational form can yield second-order pressure approximation in more general domains is still an open question. In fact, to the best of the authors' knowledge, there is no rigorous analysis available yet in the literature for this type of algorithms in general domains.

The aim of this paper is to provide a rigorous stability and error analysis for the rotational form of pressure-correction schemes. Our results indicate that while the rotational form of pressure-correction schemes does not improve the accuracy on the approximate velocity in the $L^{2}$-norm, it does improve the accuracy on this quantity in the $H^{1}$-norm and that on the approximate pressure in the $L^{2}$-norm from first-order to $\frac{3}{2}$-order. Based on our numerical results, this $\frac{3}{2}$-order convergence rate on the pressure appears to be the best possible for the rotational form of pressurecorrection schemes in general domains.

This paper is organized as follows. In 2 , we introduce notation and recall important results that are used repeatedly in the core of the paper. In 3 , we present the rotational form of the pressure-correction algorithm using a secondorder backward difference formula (BDF2) to march in time. In this section, we also analyze a singular perturbation of the Navier-Stokes equations that mimics the characteristics of the new scheme. The analysis of the discrete scheme is performed in $\$ 4$ We illustrate the performance of the proposed scheme in \$5 by showing numerical convergence tests using $\mathbb{P}_{2} / \mathbb{P}_{1}$ finite elements and $\mathbb{P}_{N} / \mathbb{P}_{N-2}$ spectral approximations.

\section{NOTATION AND PRELIMINARIES}

We now introduce some notation. We shall consider the time-dependent NavierStokes equations on a finite time interval $[0, T]$ and in an open, connected, and bounded domain $\Omega \subset \mathbb{R}^{d}(d=2$ or 3$)$ with a boundary $\Gamma$ sufficiently smooth. Let $\delta t>0$ be a time step and set $t_{k}=k \delta t$ for $0 \leq k \leq K=[T / \delta t]$.

Let $\phi^{0}, \phi^{1}, \ldots, \phi^{K}$ be some sequence of functions in some Hilbert space $E$. We denote by $\phi_{\delta t}$ this sequence, and we use the following discrete norms:

$$
\left\|\phi_{\delta t}\right\|_{l^{2}(E)}:=\left(\delta t \sum_{k=0}^{K}\left\|\phi^{k}\right\|_{E}^{2}\right)^{1 / 2}, \quad\left\|\phi_{\delta t}\right\|_{l^{\infty}(E)}:=\max _{0 \leq k \leq K}\left(\left\|\phi^{k}\right\|_{E}\right) .
$$

We use the standard Sobolev spaces $H^{m}(\Omega)(m=0, \pm 1, \cdots)$ whose norms are denoted by $\|\cdot\|_{m}$. In particular, the norm and inner product of $L^{2}(\Omega)=H^{0}(\Omega)$ are denoted by $\|\cdot\|$ and $(\cdot, \cdot)$, respectively. We also set $L_{0}^{2}(\Omega)=\left\{q \in L^{2}(\Omega): \int_{\Omega} q d x=\right.$ $0\}$. To account for homogeneous Dirichlet boundary conditions, we define $H_{0}^{1}(\Omega)=$ $\left\{v \in H^{1}(\Omega):\left.v\right|_{\Gamma}=0\right\}$. Thanks to the Poincaré inequality, for $v \in H_{0}^{1}(\Omega)^{d},\|\nabla v\|$ is a norm equivalent to $\|v\|_{1}$. We also have

$$
\|\nabla v\|^{2}=\|\nabla \cdot v\|^{2}+\|\nabla \times v\|^{2}, \forall v \in H_{0}^{1}(\Omega)^{d} .
$$


We introduce two spaces of incompressible vector fields

$$
\begin{aligned}
& H=\left\{v \in L^{2}(\Omega)^{d} ; \nabla \cdot v=0 ;\left.v \cdot n\right|_{\Gamma}=0\right\}, \\
& V=\left\{v \in H^{1}(\Omega)^{d} ; \nabla \cdot v=0 ;\left.v\right|_{\Gamma}=0\right\},
\end{aligned}
$$

and we define $P_{H}$ as the $L^{2}$-orthogonal projector in $H$, i.e.,

$$
\left(u-P_{H} u, v\right)=0 \quad \forall u \in L^{2}(\Omega)^{d}, v \in H .
$$

We denote by $c$ a generic constant that is independent of $\varepsilon$ and $\delta t$ but possibly depends on the data and the solution. We shall use the expression $A \lesssim B$ to say that there exists a generic constant $c$ such that $A \leq c B$.

Next, we define the inverse Stokes operator $S: H^{-1}(\Omega)^{d} \longrightarrow V$. For all $v$ in $H^{-1}(\Omega)^{d},(S(v), r) \in V \times L_{0}^{2}(\Omega)$ is the solution to the following problem

$$
\left\{\begin{array}{l}
(\nabla S(v), \nabla w)-(r, \nabla \cdot w)=\langle v, w\rangle, \quad \forall w \in H_{0}^{1}(\Omega)^{d}, \\
(q, \nabla \cdot S(v))=0, \quad \forall q \in L_{0}^{2}(\Omega),
\end{array}\right.
$$

where $\langle\cdot, \cdot\rangle$ denotes the duality paring between $H^{-1}(\Omega)^{d}$ and $H_{0}^{1}(\Omega)^{d}$.

We assume that $\Gamma$ is sufficiently smooth so that the following regularity properties hold ( $c f .[18])$ :

$$
\forall v \in L^{2}(\Omega)^{d}, \quad\|S(v)\|_{2}+\|\nabla r\| \leq c\|v\| .
$$

The following properties of $S$ are proved in 8 .

Lemma 2.1. For all $v$ in $H_{0}^{1}(\Omega)^{d}$ and all $0<\gamma<1$ we have

$$
(\nabla S(v), \nabla v) \geq(1-\gamma)\|v\|^{2}-c(\gamma)\left\|v-v^{\star}\right\|^{2}, \forall v^{\star} \in H .
$$

In particular,

$$
(\nabla S(v), \nabla v)=\|v\|^{2}, \forall v \in V .
$$

Lemma 2.2. The bilinear form $H^{-1}(\Omega)^{d} \times H^{-1}(\Omega)^{d} \ni(v, w) \longmapsto\langle S(v), w\rangle \in \mathbb{R}$ induces a semi-norm on $H^{-1}(\Omega)^{d}$ that we denote by $|\cdot|_{\star}$, and

$$
|v|_{\star}=\|S(v)\|_{1} \leq c\|v\|_{-1}, \forall v \in H^{-1}(\Omega)^{d} .
$$

\section{Rotational FORM OF THE PRESSURE-CORRECTION METHODS}

We consider the movement of an incompressible fluid inside $\Omega$ whose velocity $u$ and pressure $p$ are governed by the Navier-Stokes equations:

$$
\left\{\begin{array}{l}
\partial_{t} u-\nu \nabla^{2} u+u \cdot \nabla u+\nabla p=f \quad \text { in } \Omega \times[0, T], \\
\nabla \cdot u=0 \quad \text { in } \Omega \times[0, T], \\
\left.u\right|_{\Gamma}=0, u_{\mid t=0}=u_{0} \quad \text { in } \Omega .
\end{array}\right.
$$

The boundary condition on the velocity is set to zero for the sake of simplicity, and $u_{0} \in H$ is an initial velocity field.

Since for projection methods the treatment of the nonlinear term does not contribute in any essential way to the error behaviors, we shall describe the rotational pressure-correction scheme and carry out all the error analyses for the linearized equations only, thus avoiding technicalities associated with the nonlinearities which obscure the essential difficulties. In fact, the error estimates established here for the linearized equations are valid for the fully nonlinear Navier-Stokes equations, provided the solution is sufficiently smooth, and these estimates can be proved by combining the techniques used here and those in [7, 15, 17]. In practice, the nonlinear terms can be treated either implicitly, semi-implicitly or explicitly depending on 
various factors such as stability, simplicity, efficiency, and the practitioners' preferences. Thus, to fix the ideas, we will only consider the approximation of the following linearized Navier-Stokes equations (we set $\nu=1$ for simplicity):

$$
\left\{\begin{array}{l}
\partial_{t} u-\nabla^{2} u+\nabla p=f \quad \text { in } \Omega \times[0, T] \\
\nabla \cdot u=0 \quad \text { in } \Omega \times[0, T] \\
\left.u\right|_{\Gamma}=0, u_{\mid t=0}=u_{0} \quad \text { in } \Omega
\end{array}\right.
$$

To simplify our presentation, we assume that the unique solution $(u, p)$ to the above system is sufficiently smooth in time and in space.

3.1. Description of the scheme. Before introducing the rotational form of the pressure-correction algorithm, let us recall its standard form using BDF2 to march in time. Using the linearized version of the Navier-Stokes equations, the first substep accounting for viscous diffusion is

$$
\left\{\begin{array}{l}
\frac{3 \tilde{u}^{k+1}-4 u^{k}+u^{k-1}}{2 \delta t}-\nabla^{2} \tilde{u}^{k+1}+\nabla p^{k}=f\left(t_{k+1}\right), \\
\left.\tilde{u}^{k+1}\right|_{\Gamma}=0,
\end{array}\right.
$$

and the second substep accounting for incompressibility is

$$
\left\{\begin{array}{l}
\frac{3 u^{k+1}-3 \tilde{u}^{k+1}}{2 \delta t}+\nabla\left(p^{k+1}-p^{k}\right)=0, \\
\nabla \cdot u^{k+1}=0, \\
\left.u^{k+1} \cdot n\right|_{\Gamma}=0 .
\end{array}\right.
$$

This step is usually referred to as the projection step, for it is a realization of the identity $u^{k+1}=P_{H} \tilde{u}^{k+1}$.

This scheme has been thoroughly studied ( $c f$. [12, 4, 15, 7]). Though it is second-order accurate on the velocity in the $L^{2}$-norm, it is plagued by a numerical boundary layer that prevents it from being fully second-order on the $H^{1}$-norm of the velocity and on the $L^{2}$-norm of the pressure. Actually, from (3.4) we observe that $\left.\nabla\left(p^{k+1}-p^{k}\right) \cdot n\right|_{\Gamma}=0$ which implies that

$$
\left.\nabla p^{k+1} \cdot n\right|_{\Gamma}=\left.\nabla p^{k} \cdot n\right|_{\Gamma}=\left.\cdots \nabla p^{0} \cdot n\right|_{\Gamma} .
$$

It is this nonrealistic Neumann boundary condition on the pressure that introduces the numerical boundary layer referred to above and consequently limits the accuracy of the scheme.

In 1996, a modified scheme with a divergence correction has been proposed in [19]. More precisely, the second step (3.4) is replaced by

$$
\left\{\begin{array}{l}
\frac{3 u^{k+1}-3 \tilde{u}^{k+1}}{2 \delta t}+\nabla\left(p^{k+1}-p^{k}+\nabla \cdot \tilde{u}^{k+1}\right)=0 \\
\nabla \cdot u^{k+1}=0 \\
\left.u^{k+1} \cdot n\right|_{\Gamma}=0
\end{array}\right.
$$

The authors have shown numerically that the modified scheme (3.3)-(3.6) provides significantly better approximations for the pressure. To the best of our knowledge, no rigorous analysis for the modified scheme (3.3)-(3.6) has yet been proposed in the literature. 
To understand why the modified scheme performs better, we take the sum of (3.3) and (3.6) (note from (3.6) that $\nabla \times \nabla \times \tilde{u}^{k+1}=\nabla \times \nabla \times u^{k+1}$ ), and we get

$$
\left\{\begin{array}{l}
\frac{3 u^{k+1}-4 u^{k}+u^{k-1}}{2 \delta t}+\nabla \times \nabla \times u^{k+1}+\nabla p^{k+1}=f\left(t_{k+1}\right), \\
\nabla \cdot u^{k+1}=0
\end{array}\right.
$$

with $\left.u^{k+1} \cdot n\right|_{\Gamma}=0$. We observe from (3.7) that

$$
\left.\frac{\partial p^{k+1}}{\partial n}\right|_{\Gamma}=\left.\left(f\left(t_{k+1}\right)-\nabla \times \nabla \times u^{k+1}\right) \cdot n\right|_{\Gamma}
$$

which, unlike (3.5), is a consistent pressure boundary condition. The splitting error now manifests itself only in the form of an inexact tangential boundary condition for the velocity.

In view of (3.7), where the operator $\nabla \times \nabla \times$ plays a key role, we shall hereafter refer to the algorithm (3.3)-3.6) as the pressure-correction scheme in rotational form, and we shall refer to the original algorithm (3.3)-(3.4) as the pressure-correction scheme in standard form.

The aim of this paper is to prove stability and derive error estimates for the scheme (3.3)-(3.6).

3.2. Initialization of the scheme. Note that we need $\left(u^{0}, \tilde{u}^{0}, p^{0}\right)$ and $\left(u^{1}, \tilde{u}^{1}, p^{1}\right)$ to start the scheme (3.3)-(3.6). We set

$$
u^{0}=u_{0}, \tilde{u}^{0}=u_{0}, p^{0}=p(0),
$$

where $p(0)$ is determined from $u_{0}$ and equations (3.2). We solve $\left(u^{1}, \tilde{u}^{1}, p^{1}\right)$ from the following first-order pressure-correction projection scheme:

$$
\left\{\begin{array}{l}
\frac{\tilde{u}^{1}-u^{0}}{\delta t}-\nabla^{2} \tilde{u}^{1}+\nabla p^{0}=f\left(t_{1}\right) \\
\left.\tilde{u}^{1}\right|_{\Gamma}=0
\end{array}\right.
$$

and

$$
\left\{\begin{array}{l}
\frac{u^{1}-\tilde{u}^{1}}{\delta t}+\nabla\left(p^{1}-p^{0}\right)=0 \\
\nabla \cdot u^{1}=0 \\
\left.u^{1} \cdot n\right|_{\Gamma}=0
\end{array}\right.
$$

Let us denote $R^{1}=u_{t}(\delta t)-\frac{u(\delta t)-u(0)}{\delta t}$. The error equation corresponding to (3.9) is

$$
\begin{aligned}
& \left(u(\delta t)-\tilde{u}^{1}\right)-\delta t \Delta\left(u(\delta t)-\tilde{u}^{1}\right)=-\delta t \nabla(p(\delta t)-p(0))-\delta t R^{1}=O\left(\delta t^{2}\right), \\
& \left.\left(u(\delta t)-\tilde{u}^{1}\right)\right|_{\partial \Omega}=0 .
\end{aligned}
$$

One derives immediately from the standard PDE theory that

$$
\left\|u(\delta t)-\tilde{u}^{1}\right\|+\delta t^{\frac{1}{2}}\left\|\nabla\left(u(\delta t)-\tilde{u}^{1}\right)\right\|+\delta t\left\|\Delta\left(u(\delta t)-\tilde{u}^{1}\right)\right\| \lesssim \delta t^{2} .
$$

The error equation corresponding to (3.10) is

$$
\nabla\left(p(\delta t)-p^{1}\right)=-\frac{\left(u(\delta t)-u^{1}\right)-\left(u(\delta t)-\tilde{u}^{1}\right)}{\delta t}+\nabla(p(\delta t)-p(0)) .
$$

We derive easily from (3.13) and (3.12) that

$$
\left\|\nabla\left(p(\delta t)-p^{1}\right)\right\| \lesssim \delta t .
$$


Repeating the same above procedure for (3.3)-(3.6) with $k=1$, we obtain

$$
\begin{gathered}
\left\|u(2 \delta t)-\tilde{u}^{2}\right\|+\delta t^{\frac{1}{2}}\left\|\nabla\left(u(2 \delta t)-\tilde{u}^{2}\right)\right\|+\delta t\left\|\Delta\left(u(2 \delta t)-\tilde{u}^{2}\right)\right\| \lesssim \delta t^{2} . \\
\left\|\nabla\left(p(2 \delta t)-p^{2}\right)\right\| \lesssim \delta t .
\end{gathered}
$$

3.3. Time continuous version: a singularly perturbed PDE. Since the essential error behaviors of (3.3)-(3.6) are determined by its limiting system as $\delta t \longrightarrow 0$, it is instructive to study the following singularly perturbed system, which is obtained by eliminating $u^{k}$ from (3.3)-(3.6) and by dropping some lower-order terms as $\varepsilon \sim \delta t \rightarrow 0$ :

$$
\begin{gathered}
\partial_{t} u^{\varepsilon}-\nabla^{2} u^{\varepsilon}+\nabla p^{\varepsilon}=f,\left.\quad u^{\varepsilon}\right|_{\Gamma}=0, \\
\nabla \cdot u^{\varepsilon}-\varepsilon \nabla^{2} \phi^{\varepsilon}=0,\left.\frac{\partial \phi^{\varepsilon}}{\partial n}\right|_{\Gamma}=0, \\
\varepsilon \partial_{t} p^{\varepsilon}=\phi^{\varepsilon}-\nabla \cdot u^{\varepsilon},
\end{gathered}
$$

with $\left.u^{\varepsilon}\right|_{t=0}=u(0)$ and $p^{\varepsilon}(0)=p(0)$.

The following lemma exhibits the essential feature of this singularly perturbed system and is the key to prove higher order estimates.

Lemma 3.1. Provided that $u$ and $p$ are smooth enough in time and space, we have

$$
\left\|\nabla \cdot u^{\varepsilon}\right\|_{L^{\infty}\left(L^{2}(\Omega)\right)} \lesssim \varepsilon^{\frac{3}{2}} .
$$

Proof. We shall first derive some a priori estimates.

We denote $e=u^{\varepsilon}-u$ and $q=p^{\varepsilon}-p$. Subtracting (3.17) from (3.2), we find

$$
\begin{gathered}
e_{t}-\nabla^{2} e+\nabla q=0,\left.e\right|_{\Gamma}=0, \\
\nabla \cdot e-\varepsilon \nabla^{2} \phi^{\varepsilon}=0,\left.\frac{\partial \phi^{\varepsilon}}{\partial n}\right|_{\Gamma}=0, \\
\varepsilon q_{t}=\phi^{\varepsilon}-\nabla \cdot u^{\varepsilon}-\varepsilon p_{t},
\end{gathered}
$$

with $e(0)=0$ and $q(0)=0$.

Taking the inner product of the time derivative of (3.20) with $e_{t}$, we find

$$
\frac{1}{2} \partial_{t}\left\|e_{t}\right\|^{2}+\left\|\nabla e_{t}\right\|^{2}-\left(q_{t}, \nabla \cdot e_{t}\right)=0
$$

Using (3.21) and (3.22),

$$
\begin{aligned}
-\left(q_{t}, \nabla \cdot e_{t}\right) & =-\varepsilon\left(q_{t}, \Delta \phi_{t}^{\varepsilon}\right)=-\left(\phi^{\varepsilon}-\varepsilon \Delta \phi^{\varepsilon}-\varepsilon p_{t}, \Delta \phi_{t}^{\varepsilon}\right) \\
& =\frac{1}{2} \partial_{t}\left\|\nabla \phi^{\varepsilon}\right\|^{2}+\frac{\varepsilon}{2} \partial_{t}\left\|\Delta \phi^{\varepsilon}\right\|^{2}-\varepsilon\left(\nabla p_{t}, \nabla \phi_{t}^{\varepsilon}\right) \\
& =\frac{1}{2} \partial_{t}\left\|\nabla \phi^{\varepsilon}\right\|^{2}+\frac{\varepsilon}{2} \partial_{t}\left\|\Delta \phi^{\varepsilon}\right\|^{2}-\varepsilon \partial_{t}\left(\nabla p_{t}, \nabla \phi^{\varepsilon}\right)+\varepsilon\left(\nabla p_{t t}, \nabla \phi^{\varepsilon}\right) .
\end{aligned}
$$

The above two relations lead to

$$
\begin{aligned}
\frac{1}{2} \partial_{t}\left\|e_{t}\right\|^{2}+\left\|\nabla e_{t}\right\|^{2} & +\frac{1}{2} \partial_{t}\left\|\nabla \phi^{\varepsilon}\right\|^{2}+\frac{\varepsilon}{2} \partial_{t}\left\|\Delta \phi^{\varepsilon}\right\|^{2} \\
& =\varepsilon \partial_{t}\left(\nabla p_{t}, \nabla \phi^{\varepsilon}\right)-\varepsilon\left(\nabla p_{t t}, \nabla \phi^{\varepsilon}\right),
\end{aligned}
$$

since we have $e(0)=0$ and $q(0)=0$, which imply that $\phi^{\varepsilon}(0)=0$ and $e_{t}(0)=0$. Therefore, an application of the Gronwall lemma to the above relation leads to

$$
\left\|e_{t}(t)\right\|^{2}+\left\|\nabla \phi^{\varepsilon}(t)\right\|^{2}+\varepsilon\left\|\Delta \phi^{\varepsilon}(t)\right\|^{2}+\int_{0}^{t}\left\|\nabla e_{t}(s)\right\|^{2} d s \lesssim \varepsilon^{2} .
$$


We immediately obtain

$$
\left\|\nabla \cdot u^{\varepsilon}(t)\right\|^{2}=\varepsilon^{2}\left\|\Delta \phi^{\varepsilon}(t)\right\|^{2} \lesssim \varepsilon^{3} .
$$

Lemma 3.2. Provided that $u$ and $p$ are smooth enough in time and space, we have

$$
\left\|u-u^{\varepsilon}\right\|_{L^{2}\left(L^{2}(\Omega)^{d}\right)} \lesssim \varepsilon^{2} .
$$

Proof. We take the inner product of (3.20) with $S(e)$, where $S$ is the inverse Stokes operator defined in $\S 2$. Since $S(e) \in V$, we find

$$
\frac{1}{2} \partial_{t}|e|_{\star}^{2}+(\nabla e, \nabla S(e))=0 .
$$

Thanks to Lemma 2.1, we have

$$
\frac{1}{2} \partial_{t}|e|_{\star}^{2}+\frac{1}{2}\|e\|^{2} \lesssim\left\|e-P_{H} e\right\|^{2} .
$$

By the definition of $P_{H}$, we can write $e-P_{H} e=\nabla r$ with $\left.\frac{\partial r}{\partial n}\right|_{\partial \Omega}=0$. Consequently $\nabla \cdot e=\Delta r$ and from (3.21), we infer $r=\varepsilon \phi^{\varepsilon}$ and

$$
\left\|e-P_{H} e\right\|^{2}=\|\nabla r\|^{2}=\varepsilon^{2}\left\|\nabla \phi^{\varepsilon}\right\|^{2} .
$$

Applying the Gronwall lemma to (3.29) and taking into account (3.26), we derive

$$
|e(t)|_{\star}^{2}+\int_{0}^{t}\|e(s)\|^{2} d s \lesssim \varepsilon^{2} \int_{0}^{t}\left\|\nabla \phi^{\varepsilon}(s)\right\|^{2} d s \lesssim \varepsilon^{4} .
$$

Remark 3.1. These two lemmas are essential to understanding the arguments that will be used in the discrete case. They will have two discrete counterparts in the form of Lemmas 4.1 and 4.2 ,

\section{Error estimates for the time Discrete Case}

Let us first introduce some notation. For any sequence $\phi^{0}, \phi^{1}, \ldots$, we set

$$
\delta_{t} \phi^{k}=\phi^{k}-\phi^{k-1}, \quad \delta_{t t} \phi^{k}=\delta_{t}\left(\delta_{t} \phi^{k}\right), \quad \delta_{t t t} \phi^{k}=\delta_{t}\left(\delta_{t t} \phi^{k}\right)
$$

and

$$
\begin{cases}e^{k}=u\left(t_{k}\right)-u^{k}, & \tilde{e}^{k}=u\left(t_{k}\right)-\tilde{u}^{k} \\ \psi^{k}=p\left(t_{k+1}\right)-p^{k}, & q^{k}=p\left(t_{k}\right)-p^{k}\end{cases}
$$

The main result in this paper is

Theorem 4.1. Provided the solution to (3.2) is smooth enough in time and space, the solution $\left(u^{k}, \tilde{u}^{k}, p^{k}\right)$ to (3.3)-(3.6) satisfies the estimates

$$
\begin{aligned}
&\left\|e_{\delta t}\right\|_{l^{2}\left(L^{2}(\Omega)^{d}\right)}+\left\|\tilde{e}_{\delta t}\right\|_{l^{2}\left(L^{2}(\Omega)^{d}\right)} \lesssim \delta t^{2}, \\
&\left\|e_{\delta t}\right\|_{l^{2}\left(H^{1}(\Omega)^{d}\right)}+\left\|\tilde{e}_{\delta t}\right\|_{l^{2}\left(H^{1}(\Omega)^{d}\right)}+\left\|q_{\delta t}\right\|_{l^{2}\left(L^{2}(\Omega)\right)} \lesssim \delta t^{\frac{3}{2}} .
\end{aligned}
$$

The remainder of this section is devoted to the proof of the above theorem. The proof of Theorem 4.1 will be carried out through a sequence of estimates presented below. 
4.1. Stability and a priori estimate on $\left\|\nabla \cdot \tilde{u}^{k}\right\|$. We first establish a result similar to that of Lemma 3.1

Lemma 4.1. Under the hypotheses of Theorem 4.1, we have

$$
\begin{aligned}
\left\|\nabla \cdot \tilde{u}_{\delta t}\right\|_{l^{\infty}\left(L^{2}(\Omega)\right)} & \lesssim \delta t^{3 / 2}, \\
\left\|e_{\delta t}-\tilde{e}_{\delta t}\right\|_{\ell^{2}\left(L^{2}(\Omega)^{d}\right)} & \lesssim \delta t^{2}, \\
\left\|\delta_{t} e_{\delta t}-\delta_{t} \tilde{e}_{\delta t}\right\|_{l^{2}\left(L^{2}(\Omega)^{d}\right)} & \lesssim \delta t^{5 / 2} .
\end{aligned}
$$

Proof. The proof of this lemma follows the same principle as that we used in $\S 3$ for the time continuous version of the algorithm. The critical step here consists in working with the time increments $\delta_{t} e^{k+1}$ and $\delta_{t} \tilde{e}^{k+1}$, which corresponds to taking the inner product of the time derivative of (3.20) with $\partial_{t} e$.

Let us first write the equations that control the time increments of the errors. We define

$$
R^{k}=\frac{3 u\left(t_{k}\right)-4 u\left(t_{k-1}\right)+u\left(t_{k-2}\right)}{2 \delta t}-\partial_{t} u\left(t_{k}\right)
$$

Then, for $k \geq 1$, we have

$$
\left\{\begin{array}{l}
\frac{3 \delta_{t} \tilde{e}^{k+1}-4 \delta_{t} e^{k}+\delta_{t} e^{k-1}}{2 \delta t}-\Delta \delta_{t} \tilde{e}^{k+1}+\nabla \delta_{t} \psi^{k}=\delta_{t} R^{k+1}, \\
\left.\delta_{t} \tilde{e}^{k+1}\right|_{\Gamma}=0
\end{array}\right.
$$

and

$$
\left\{\begin{array}{l}
\frac{3}{2 \delta t} \delta_{t} e^{k+1}+\nabla\left(\delta_{t} q^{k+1}+\nabla \cdot \tilde{e}^{k+1}\right)=\frac{3}{2 \delta t} \delta_{t} \tilde{e}^{k+1}+\nabla\left(\delta_{t} \psi^{k}+\nabla \cdot \tilde{e}^{k}\right), \\
\left.\delta_{t} e^{k+1} \cdot n\right|_{\Gamma}=0 .
\end{array}\right.
$$

We take the inner product of (4.3) with $4 \delta t \delta_{t} \tilde{e}^{k+1}$ to get

$$
\begin{aligned}
2\left(\delta_{t} \tilde{e}^{k+1}, 3 \delta_{t} \tilde{e}^{k+1}\right. & \left.-4 \delta_{t} e^{k}+\delta_{t} e^{k-1}\right)+4 \delta t\left\|\nabla \delta_{t} \tilde{e}^{k+1}\right\|^{2}+4 \delta t\left(\delta_{t} \tilde{e}^{k+1}, \nabla \delta_{t} \psi^{k}\right) \\
& =4 \delta t\left(\delta_{t} e^{k+1}, \delta_{t} R^{k+1}\right) \leq 2 \delta t\left\|\delta_{t} e^{k+1}\right\|^{2}+2 \delta t\left\|\delta_{t} R^{k+1}\right\|^{2}
\end{aligned}
$$

The treatment of the first term is quite technical but similar to that in [8]. For the readers' convenience, we show the details below. We denote

$$
\begin{aligned}
I & =2\left(\delta_{t} \tilde{e}^{k+1}, 3 \delta_{t} \tilde{e}^{k+1}-4 \delta_{t} e^{k}+\delta_{t} e^{k-1}\right) \\
& =6\left(\delta_{t} \tilde{e}^{k+1}, \delta_{t} \tilde{e}^{k+1}-\delta_{t} e^{k+1}\right)+2\left(\delta_{t} \tilde{e}^{k+1}-\delta_{t} e^{k+1}, 3 \delta_{t} e^{k+1}-4 \delta_{t} e^{k}+\delta_{t} e^{k-1}\right) \\
& +2\left(\delta_{t} e^{k+1}, 3 \delta_{t} e^{k+1}-4 \delta_{t} e^{k}+\delta_{t} e^{k-1}\right),
\end{aligned}
$$

and we denote by $I_{1}, I_{2}$ and $I_{3}$ the three terms in the right-hand side. Using the algebraic identities

$$
\begin{aligned}
2\left(a^{k+1}, a^{k+1}-a^{k}\right)= & \left|a^{k+1}\right|^{2}+\left|a^{k+1}-a^{k}\right|^{2}-\left|a^{k}\right|^{2} \\
2\left(a^{k+1}, 3 a^{k+1}-4 a^{k}+a^{k-1}\right)= & \left|a^{k+1}\right|^{2}+\left|2 a^{k+1}-a^{k}\right|^{2}+\left|\delta_{t t} a^{k+1}\right|^{2} \\
& -\left|a^{k}\right|^{2}-\left|2 a^{k}-a^{k-1}\right|^{2}
\end{aligned}
$$

we derive

$$
\begin{aligned}
& I_{1}=3\left\|\delta_{t} \tilde{e}^{k+1}\right\|^{2}+3\left\|\delta_{t} e^{k+1}-\delta_{t} \tilde{e}^{k+1}\right\|^{2}-3\left\|\delta_{t} e^{k+1}\right\|^{2}, \\
& I_{3}=\left\|\delta_{t} e^{k+1}\right\|^{2}+\left\|2 \delta_{t} e^{k+1}-\delta_{t} e^{k}\right\|^{2}+\left\|\delta_{t t t} e^{k+1}\right\|^{2}-\left\|\delta_{t} e^{k}\right\|^{2}-\left\|2 \delta_{t} e^{k}-\delta_{t} e^{k-1}\right\|^{2} .
\end{aligned}
$$


Due to (4.4) and using the fact that $e^{k} \in H$, we derive the equality

$$
\frac{3}{2 \delta t} I_{2}=2\left(\nabla \delta_{t}\left(q^{k+1}-\psi^{k}\right)+\nabla \nabla \cdot \delta_{t} \tilde{e}^{k+1}, 3 \delta_{t} e^{k+1}-4 \delta_{t} e^{k}+\delta_{t} e^{k-1}\right)=0 .
$$

Combining all the above results, we obtain

$$
\begin{aligned}
3\left\|\delta_{t} \tilde{e}^{k+1}\right\|^{2}- & 3\left\|\delta_{t} e^{k+1}\right\|^{2}+3\left\|\delta_{t} e^{k+1}-\delta_{t} \tilde{e}^{k+1}\right\|^{2}+\left\|\delta_{t} e^{k+1}\right\|^{2} \\
& +\left\|2 \delta_{t} e^{k+1}-\delta_{t} e^{k}\right\|^{2}-\left\|\delta_{t} e^{k}\right\|^{2}-\left\|2 \delta_{t} e^{k}-\delta_{t} e^{k-1}\right\|^{2}+\left\|\delta_{t t t} e^{k+1}\right\|^{2} \\
& +4 \delta t\left\|\nabla \delta_{t} \tilde{e}^{k+1}\right\|^{2}+4 \delta t\left(\delta_{t} \tilde{e}^{k+1}, \nabla \delta_{t} \psi^{k}\right)=4 \delta t\left(\delta_{t} e^{k+1}, \delta_{t} R^{k+1}\right) .
\end{aligned}
$$

By taking the square of (4.4), multiplying the result by $\frac{4}{3} \delta t^{2}$ and integrating over the domain, we have

$$
\begin{aligned}
3\left\|\delta_{t} e^{k+1}\right\|^{2} & +\frac{4 \delta t^{2}}{3}\left\|\nabla\left(\delta_{t} q^{k+1}+\nabla \cdot \tilde{e}^{k+1}\right)\right\|^{2} \\
& =3\left\|\delta_{t} \tilde{e}^{k+1}\right\|^{2} \\
& +4 \delta t\left(\delta_{t} \tilde{e}^{k+1}, \nabla\left(\delta_{t} \psi^{k}+\nabla \cdot \tilde{e}^{k}\right)\right)+\frac{4 \delta t^{2}}{3}\left\|\nabla\left(\delta_{t} \psi^{k}+\nabla \cdot \tilde{e}^{k}\right)\right\|^{2} .
\end{aligned}
$$

The last two terms in the above relation can be bounded as follows.

The term $4 \delta t\left(\delta_{t} \tilde{e}^{k+1}, \nabla \delta_{t} \psi^{k}\right)$ cancels out with the same term in (4.8). Integrating by parts and using (4.6), we infer

$$
\begin{aligned}
4 \delta t\left(\delta_{t} \tilde{e}^{k+1}, \nabla \nabla \cdot \tilde{e}^{k}\right) & =-4 \delta t\left(\nabla \cdot\left(\tilde{e}^{k+1}-\tilde{e}^{k}\right), \nabla \cdot \tilde{e}^{k}\right) \\
& =2 \delta t\left(\left\|\nabla \cdot \tilde{e}^{k}\right\|^{2}-\left\|\nabla \cdot \tilde{e}^{k+1}\right\|^{2}+\left\|\nabla \cdot \delta_{t} \tilde{e}^{k+1}\right\|^{2}\right) .
\end{aligned}
$$

The term $2 \delta t\left\|\nabla \cdot \delta_{t} \tilde{e}^{k+1}\right\|^{2}$ can be controlled by $4 \delta t\left\|\nabla \delta_{t} \tilde{e}^{k+1}\right\|^{2}$ in (4.8), thanks to the identity (2.2), we have

$$
\left\|\nabla \delta_{t} \tilde{e}^{k+1}\right\|^{2}=\left\|\nabla \times \delta_{t} \tilde{e}^{k+1}\right\|^{2}+\left\|\nabla \cdot \delta_{t} \tilde{e}^{k+1}\right\|^{2} .
$$

For $\left\|\nabla\left(\delta_{t} \psi^{k}+\nabla \cdot \tilde{e}^{k}\right)\right\|^{2}$, we have

$$
\begin{aligned}
\left\|\nabla\left(\delta_{t} \psi^{k}+\nabla \cdot \tilde{e}^{k}\right)\right\|^{2} & =\left\|\nabla\left(\delta_{t} q^{k}+\nabla \cdot \tilde{e}^{k}\right)+\nabla \delta_{t t} p\left(t_{k+1}\right)\right\|^{2} \\
& \leq\left(c \delta t^{2}+\left\|\nabla\left(\delta_{t} q^{k}+\nabla \cdot \tilde{e}^{k}\right)\right\|\right)^{2} \\
& \leq c \delta t^{4}+2 c \delta t^{2}\left\|\nabla\left(\delta_{t} q^{k}+\nabla \cdot \tilde{e}^{k}\right)\right\|+\left\|\nabla\left(\delta_{t} q^{k}+\nabla \cdot \tilde{e}^{k}\right)\right\|^{2} \\
& \leq c \delta t^{4}+c \delta t\left(\delta t^{2}+\left\|\nabla\left(\delta_{t} q^{k}+\nabla \cdot \tilde{e}^{k}\right)\right\|^{2}\right)+\left\|\nabla\left(\delta_{t} q^{k}+\nabla \cdot \tilde{e}^{k}\right)\right\|^{2} \\
& \leq c \delta t^{3}+(1+c \delta t)\left\|\nabla\left(\delta_{t} q^{k}+\nabla \cdot \tilde{e}^{k}\right)\right\|^{2} .
\end{aligned}
$$

Combining the relations (4.8)-4.12), we derive

$$
\begin{aligned}
& 3\left\|\delta_{t}\left(e^{k+1}-\tilde{e}^{k+1}\right)\right\|^{2}+\left\|\delta_{t} e^{k+1}\right\|^{2}+\left\|2 \delta_{t} e^{k+1}-\delta_{t} e^{k}\right\|^{2}+\left\|\delta_{t t t} e^{k+1}\right\|^{2} \\
& \quad+2 \delta t\left\|\nabla \delta_{t} \tilde{e}^{k+1}\right\|^{2}+2 \delta t\left\|\nabla \cdot \tilde{e}^{k+1}\right\|^{2}+\frac{4}{3} \delta t^{2}\left\|\nabla\left(\delta_{t} q^{k+1}+\nabla \cdot \tilde{e}^{k+1}\right)\right\|^{2} \\
& \leq c \delta t^{5}+2 \delta t\left\|\delta_{t} e^{k+1}\right\|^{2}+(1+c \delta t) \frac{4}{3} \delta t^{2}\left\|\nabla\left(\delta_{t} q^{k}+\nabla \cdot \tilde{e}^{k}\right)\right\|^{2} \\
&+\left\|\delta_{t} e^{k}\right\|^{2}+\left\|2 \delta_{t} e^{k}-\delta_{t} e^{k-1}\right\|^{2}+2 \delta t\left\|\nabla \cdot \tilde{e}^{k}\right\|^{2}+2 \delta t\left\|\delta_{t} R^{k+1}\right\|^{2} .
\end{aligned}
$$


Finally, applying the discrete Gronwall lemma to the above relation and taking into account the initial estimates (3.12) $-($ (3.16) $)$, we obtain

$$
\begin{aligned}
\delta t\left\|\nabla \cdot \tilde{e}^{k+1}\right\|^{2}+\left\|\delta_{t} e^{k+1}\right\|^{2}+\left\|2 \delta_{t} e^{k+1}-\delta_{t} e^{k}\right\|^{2}+\sum_{l=2}^{k}\left\|\delta_{t} e^{l+1}-\delta_{t} \tilde{e}^{l+1}\right\|^{2} \\
\quad+\delta t \sum_{l=2}^{k}\left\|\nabla \delta_{t} \tilde{e}^{l+1}\right\|^{2}+\delta t^{2}\left\|\nabla\left(\delta_{t} q^{k+1}+\nabla \cdot \tilde{e}^{k+1}\right)\right\|^{2} \\
\leq c\left(\delta t^{4}+\delta t\left\|\nabla \cdot \tilde{e}^{2}\right\|^{2}+\left\|\delta_{t} e^{2}\right\|^{2}+\left\|2 \delta_{t} e^{2}-\delta_{t} e^{1}\right\|^{2}+\delta t^{2}\left\|\nabla\left(\delta_{t} q^{2}+\nabla \cdot \tilde{e}^{2}\right)\right\|^{2}\right) \\
\quad+c \delta t \sum_{l=2}^{k}\left\|\delta_{t} R^{l+1}\right\|^{2} \\
\leq c \delta t^{4}+\delta t \sum_{l=2}^{k}\left\|\delta_{t} R^{l+1}\right\|^{2} \lesssim \delta t^{4} .
\end{aligned}
$$

The estimates on $\nabla \cdot \tilde{u}_{\delta t}$ and $\delta_{t} e_{\delta t}-\delta_{t} \tilde{e}_{\delta t}$ are now direct consequences of the above inequality. To derive the estimate on $e_{\delta t}-\tilde{e}_{\delta t}$, we use (3.6) to infer

$$
e^{k+1}-\tilde{e}^{k+1}=-\frac{2 \delta t}{3} \nabla\left(\delta_{t} q^{k+1}+\nabla \cdot \tilde{e}^{k+1}-\delta_{t} p\left(t_{k+1}\right)\right) .
$$

Then, it is clear that

$$
\left\|e_{\delta t}-\tilde{e}_{\delta t}\right\|_{\ell^{2}\left(L^{2}(\Omega)^{d}\right)} \lesssim \delta t\left\|\nabla\left(\delta_{t} q_{\delta t}+\nabla \cdot \tilde{e}_{\delta t}\right)\right\|_{\ell^{2}\left(L^{2}(\Omega)^{d}\right)}+\delta t^{2} \lesssim \delta t^{2} .
$$

Remark 4.1. From (4.13), we observe the remarkable fact that the bound $\left\|\nabla \cdot \tilde{u}_{\delta t}\right\|_{l^{2}\left(L^{2}(\Omega)\right)} \leq \delta t^{3 / 2}$ still holds even if we replace the second-order BDF2 time stepping in (3.3) and (3.6) by the first-order backward Euler time stepping (i.e., in the case $\left.R^{k+1} \sim O(\delta t)\right)$.

4.2. Error estimates. We start with a result similar to that stated in Lemma 3.2

Lemma 4.2. Under the hypotheses of Theorem 4.1, we have

$$
\left\|\tilde{e}_{\delta t}\right\|_{l^{2}\left(L^{2}(\Omega)^{d}\right)} \lesssim \delta t^{2} .
$$

Proof. From (3.6), we can write

$$
u^{k+1}=\tilde{u}^{k+1}-\frac{2 \delta t}{3} \nabla\left(p^{k+1}-p^{k}+\nabla \cdot \tilde{u}^{k+1}\right) .
$$

Substituting the above relation in (3.3) and considering the error equation, we obtain

$$
\frac{3 \tilde{e}^{k+1}-4 \tilde{e}^{k}+\tilde{e}^{k-1}}{2 \delta t}-\nabla^{2} \tilde{e}^{k+1}+\nabla \gamma^{k+1}=R^{k+1},
$$

where $\nabla \gamma^{k+1}$ represents all the gradient terms in the error equation and $R^{k+1}$ is defined in (4.2).

As in the time continuous case, we make use of the inverse Stokes operator. By taking the inner product of (4.15) with $4 \delta t S\left(\tilde{e}^{k+1}\right)$ and using the identity (4.7), we 
obtain

$$
\begin{aligned}
\left|\tilde{e}^{k+1}\right|_{\star}^{2}+\left|2 \tilde{e}^{k+1}-\tilde{e}^{k}\right|_{\star}^{2}+\left|\delta_{t t} \tilde{e}^{k+1}\right|_{\star}^{2}+4 \delta t\left(\nabla S\left(\tilde{e}^{k+1}\right), \nabla \tilde{e}^{k+1}\right) \\
=4 \delta t\left(R^{k+1}, S\left(\tilde{e}^{k+1}\right)\right)+\left|\tilde{e}^{k}\right|_{\star}^{2}+\left|2 \tilde{e}^{k}-\tilde{e}^{k-1}\right|_{\star}^{2} .
\end{aligned}
$$

Thanks to Lemma 2.1 and the fact that $e^{k+1}$ is in $H$, we deduce

$$
4 \delta t\left(\nabla S\left(\tilde{e}^{k+1}\right), \nabla \tilde{e}^{k+1}\right) \geq 2 \delta t\left\|\tilde{e}^{k+1}\right\|^{2}-c \delta t\left\|\tilde{e}^{k+1}-e^{k+1}\right\|^{2} .
$$

Due to (2.6), we have

$$
4 \delta t\left(R^{k+1}, S\left(\tilde{e}^{k+1}\right)\right) \leq c \delta t\left\|R^{k+1}\right\|_{-1}^{2}+\delta t\left\|\tilde{e}^{k+1}\right\|^{2} .
$$

As a result, we obtain

$$
\begin{aligned}
& \left|\tilde{e}^{k+1}\right|_{\star}^{2}+\left|2 \tilde{e}^{k+1}-\tilde{e}^{k}\right|_{\star}^{2}+\left|\delta_{t t} \tilde{e}^{k+1}\right|_{\star}^{2}+\delta t\left\|\tilde{e}^{k+1}\right\|^{2} \\
& \quad \leq c \delta t\left\|R^{k+1}\right\|_{-1}^{2}+c \delta t\left\|\tilde{e}^{k+1}-e^{k+1}\right\|^{2}+\left|\tilde{e}^{k}\right|_{\star}^{2}+\left|2 \tilde{e}^{k}-\tilde{e}^{k-1}\right|_{\star}^{2} .
\end{aligned}
$$

Applying the discrete Gronwall lemma and using the initial estimates (3.12), (3.15) and Lemma 2.2, we infer

$$
\left\|\tilde{e}_{\delta t}\right\|_{l^{2}\left(L^{2}(\Omega)^{d}\right)}^{2} \leq c\left\|\tilde{e}_{\delta t}-e_{\delta t}\right\|_{l^{2}\left(L^{2}(\Omega)^{d}\right)}^{2}+\delta t^{4} .
$$

The desired result is now an easy consequence of Lemma 4.1.

The key for obtaining improved estimates on $\left\|\tilde{e}_{\delta t}\right\|_{l^{2}\left(H^{1}(\Omega)^{d}\right)}$ and $\left\|q_{\delta t}\right\|_{l^{2}\left(L^{2}(\Omega)\right)}$ is to derive an improved estimate on $\frac{1}{2 \delta t}\left(3 \delta_{t} \tilde{e}^{k+1}-4 \delta_{t} \tilde{e}^{k}+\delta_{t} \tilde{e}^{k-1}\right)$.

For any sequence of functions $\phi^{0}, \phi^{1}, \ldots$, we set $\mathcal{D}_{t} \phi^{k+1}=\frac{1}{2}\left(3 \phi^{k+1}-4 \phi^{k}+\phi^{k-1}\right)$.

Lemma 4.3. Under the hypotheses of Theorem 4.1, we have

$$
\left\|\left(\mathcal{D}_{t} \tilde{e}\right)_{\delta t}\right\|_{l^{2}\left(L^{2}(\Omega)^{d}\right)} \lesssim \delta t^{5 / 2} .
$$

Proof. We use the same argument as that in the proof of the $L^{2}$-estimate, but we use it on the time increment $\delta_{t} \tilde{e}^{k+1}$. For $k \geq 2$ we have

$$
\frac{3 \delta_{t} \tilde{e}^{k+1}-4 \delta_{t} \tilde{e}^{k}+\delta_{t} \tilde{e}^{k-1}}{2 \delta t}-\nabla^{2} \delta_{t} \tilde{e}^{k+1}+\nabla \delta_{t} \gamma^{k+1}=\delta_{t} R^{k+1}
$$

By taking the inner product of the above relation with $4 \delta t S\left(\delta_{t} \tilde{e}^{k+1}\right)$ and repeating the same arguments as in the previous lemma, we obtain

$$
\begin{aligned}
\left|\delta_{t} \tilde{e}^{k+1}\right|_{\star}^{2}+\left|2 \delta_{t} \tilde{e}^{k+1}-\delta_{t} \tilde{e}^{k}\right|_{\star}^{2}+\left|\delta_{t t t} \tilde{e}^{k+1}\right|_{\star}^{2}+\delta t\left\|\delta_{t} \tilde{e}^{k+1}\right\|^{2} \\
\leq c \delta t\left\|\delta_{t} R^{k+1}\right\|^{2}+c \delta t\left\|\delta_{t} \tilde{e}^{k+1}-\delta_{t} e^{k+1}\right\|^{2}+\left|\delta_{t} \tilde{e}^{k}\right|_{\star}^{2}+\left|2 \delta_{t} \tilde{e}^{k}-\delta_{t} \tilde{e}^{k-1}\right|_{\star}^{2} .
\end{aligned}
$$

Applying the discrete Gronwall lemma and using the initial estimates (3.12), (3.15) and Lemma 4.1, we obtain

$$
\begin{aligned}
\left\|\delta_{t} \tilde{e}_{\delta t}\right\|_{l^{2}\left(L^{2}(\Omega)^{d}\right)}^{2} & \leq c\left\|\delta_{t} \tilde{e}_{\delta t}-\delta_{t} e_{\delta t}\right\|_{l^{2}\left(L^{2}(\Omega)^{d}\right)}^{2}+\left|\delta_{t} \tilde{e}^{2}\right|_{\star}^{2}+\left|2 \delta_{t} \tilde{e}^{2}-\delta_{t} \tilde{e}^{1}\right|_{\star}^{2} \\
& +c \delta t\left\|\delta_{t} R^{k+1}\right\|^{2} \lesssim \delta t^{5} .
\end{aligned}
$$

The result follows from the above estimate and the fact that $2 \mathcal{D}_{t} \tilde{e}^{k+1}=3 \delta_{t} \tilde{e}^{k+1}-$ $\delta_{t} \tilde{e}^{k}$.

We are now in position to prove the remaining claims in Theorem 4.1 .

Lemma 4.4. Under the hypotheses of Theorem 4.1, we have

$$
\left\|e_{\delta t}\right\|_{l^{2}\left(H^{1}(\Omega)^{d}\right)}+\left\|\tilde{e}_{\delta t}\right\|_{l^{2}\left(H^{1}(\Omega)^{d}\right)}+\left\|q_{\delta t}\right\|_{l^{2}\left(L^{2}(\Omega)\right)} \lesssim \delta t^{\frac{3}{2}} .
$$


Proof. We start from the reformulated relation (3.7). Using (3.6), we have

$$
\nabla \times \nabla \times u^{k+1}=\nabla \times \nabla \times \tilde{u}^{k+1}=-\nabla^{2} \tilde{u}^{k+1}+\nabla \nabla \cdot \tilde{u}^{k+1} .
$$

Thus, the error equation corresponding to (3.7) and (3.6) can be written as a nonhomogeneous Stokes system for the couple $\left(\tilde{e}^{k+1}, q^{k+1}+\nabla \cdot \tilde{e}^{k+1}\right)$ :

$$
\begin{aligned}
& -\nabla^{2} \tilde{e}^{k+1}+\nabla\left(q^{k+1}+\nabla \cdot \tilde{e}^{k+1}\right)=h^{k+1}, \\
& \nabla \cdot \tilde{e}^{k+1}=g^{k+1},\left.\tilde{e}^{k+1}\right|_{\Gamma}=0,
\end{aligned}
$$

where

$$
\begin{aligned}
& h^{k+1}=R^{k+1}-\frac{3 e^{k+1}-4 e^{k}+e^{k-1}}{2 \delta t}, \\
& g^{k+1}=-\frac{2 \delta t}{3} \nabla^{2}\left(p^{k+1}-p^{k}+\nabla \cdot \tilde{e}^{k+1}\right) .
\end{aligned}
$$

Thanks to Lemma 4.1 we have

$$
\left\|g^{k+1}\right\|=\left\|\nabla \cdot \tilde{e}^{k+1}\right\| \lesssim \delta t^{\frac{3}{2}}, \forall k .
$$

Since $e^{k}=P_{H} \tilde{e}^{k}$, we have

$$
\left\|\frac{3 e^{k+1}-4 e^{k}+e^{k-1}}{2 \delta t}\right\| \leq\left\|\frac{3 \tilde{e}^{k+1}-4 \tilde{e}^{k}+\tilde{e}^{k-1}}{2 \delta t}\right\|=\frac{1}{\delta t}\left\|\mathcal{D}_{t} \tilde{e}^{k+1}\right\| .
$$

Hence, we have

$$
\left\|h^{k+1}\right\|_{-1} \leq\left\|R^{k+1}\right\|_{-1}+\left\|\frac{3 e^{k+1}-4 e^{k}+e^{k-1}}{2 \delta t}\right\|_{-1} \leq\left\|R^{k+1}\right\|_{-1}+\frac{1}{\delta t}\left\|\mathcal{D}_{t} \tilde{e}^{k+1}\right\|_{-1} .
$$

Now, we apply the following standard stability result for nonhomogeneous Stokes systems [18] to [4.16),

$$
\left\|\tilde{e}^{k+1}\right\|_{1}+\left\|\left(q^{k+1}+\nabla \cdot \tilde{e}^{k+1}\right)\right\| \lesssim\left\|h^{k+1}\right\|_{-1}+\left\|g^{k+1}\right\| .
$$

Thanks to (4.18), (4.19) and Lemma 4.3, we derive

$$
\left\|\tilde{e}_{\delta t}\right\|_{l^{2}\left(H^{1}(\Omega)^{d}\right)} \lesssim \delta t^{\frac{3}{2}}
$$

Then, from

$$
\left\|q^{k+1}\right\| \leq\left\|q^{k+1}+\nabla \cdot \tilde{e}^{k+1}\right\|+\left\|\nabla \cdot \tilde{e}^{k+1}\right\|,
$$

we derive

$$
\left\|q_{\delta t}\right\|_{l^{2}\left(L^{2}(\Omega)\right)} \lesssim \delta t^{\frac{3}{2}} .
$$

We conclude by using the fact that $\left\|P_{H} v\right\|_{1} \lesssim\|v\|_{1}$ for all $v \in H_{0}^{1}(\Omega)^{d}$ (cf. Remark I.1.6 in 18]).

Thus, all the bounds stated in Theorem 4.1 have been proved.

\section{Numerical RESUlts AND DISCUSSIONS}

5.1. Numerical results with a spectral approximation. Let us first consider a square domain $\Omega=(-1,1)^{2}$ with Dirichlet boundary conditions on the velocity. We have implemented the second-order pressure-correction scheme in standard and rotational forms with a Legendre-Galerkin approximation [14. Denoting by $\mathbb{P}_{N}$ the space of polynomials of degree less than or equal to $N$, we approximate the velocity and the pressure in $\mathbb{P}_{N} \times \mathbb{P}_{N}$ and $\mathbb{P}_{N-2}$, respectively. 

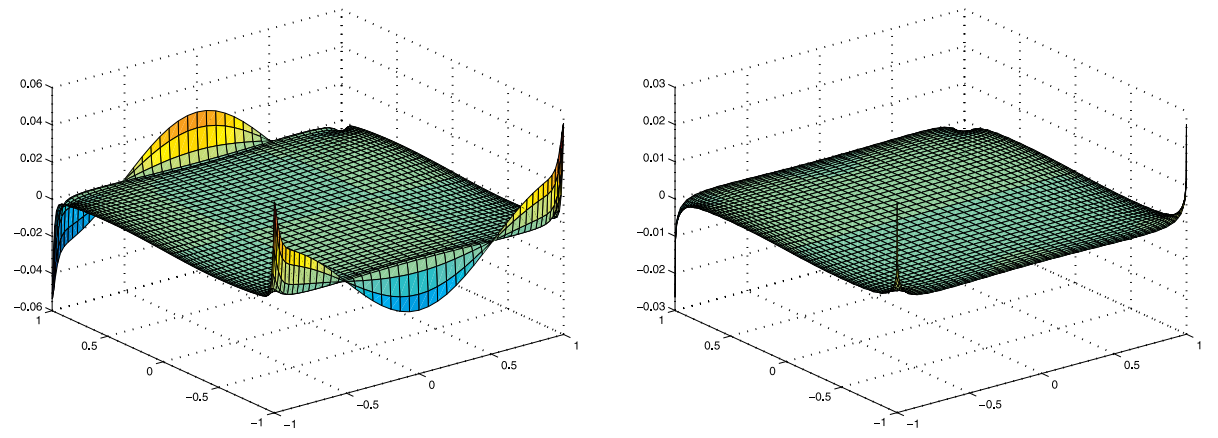

Figure 1. Pressure error field at time $t=1$ in a square: left, standard form; right, rotational form.

We take the exact solution $(u, p)$ of the linearized (at $u=0$ ) Navier-Stokes equations to be

$$
\begin{aligned}
& u(x, y, t)=\pi \sin t\left(\sin 2 \pi y \sin ^{2} \pi x,-\sin 2 \pi x \sin ^{2} \pi y\right), \\
& p(x, y, t)=\sin t \cos \pi x \sin \pi y .
\end{aligned}
$$

Then the source term $f$ is given by $f=u_{t}-\Delta u+\nabla p$. In the computations reported herein, we take $N=48$ so that the spatial discretization errors are negligible compared with the time discretization errors.

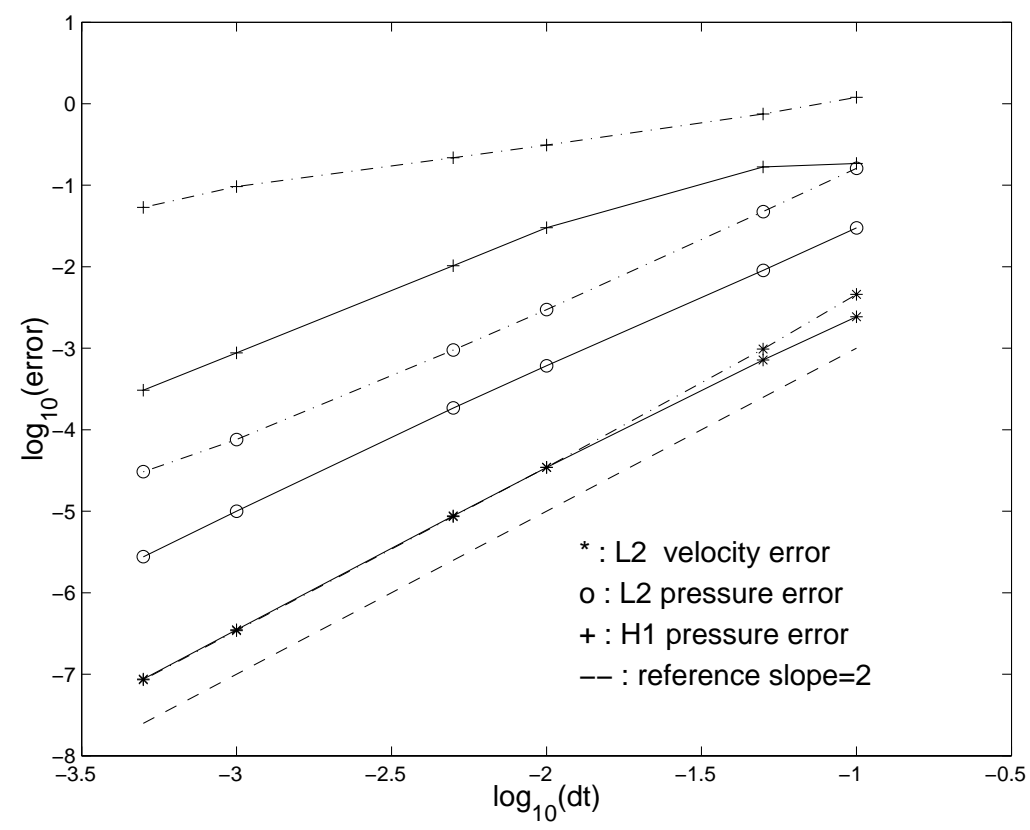

Figure 2. Convergence rates in a square: dashed lines for the standard formulation and solid lines for the rotational formulation. 
In Figure 1, we plot the pressure error field at $t=1$ for a typical time step, and in Figure 2, we represent errors on the pressure and the velocity measured in various norms as functions of the time step $\delta t$.

We note in Figure 1 that for the standard form of the algorithm, a numerical boundary layer appears on the two boundaries $\{(x, y): x \in(-1,1), y= \pm 1\}$ where the exact pressure is such that $\frac{\partial p}{\partial \vec{n}} \neq 0$ ( $\frac{\partial p}{\partial \vec{n}}=0$ on the other two boundaries). For the rotational form, there is no numerical boundary layer, but we observe large spikes at the four corners of the domain. These observations suggest that the divergence correction in the rotational form, which leads to consistent approximate pressure Neumann boundary conditions, successfully cured the numerical boundary layer problem. However, the large errors at the four corners degrade the global convergence rate of the pressure approximation. Indeed, we observe in Figure 2 that the pressure approximation from the rotational formulation is not fully secondorder accurate, though it is significantly more accurate than that calculated using the standard formulation. We also note that the rotational formulation does not yield any improvement on the approximation of the velocity in the $L^{2}$-norm.

To better understand why there are localized large errors at the corners of the domain, we have also implemented the standard and rotational forms of the pressurecorrection scheme in a periodic channel $\Omega=(0,2 \pi) \times(-1,1)$. We assume that the solution is periodic in the $x$ direction and that the velocity is subject to a Dirichlet boundary condition at $y= \pm 1$. We choose the same exact solution $(u, p)$ as given above, and we use a Fourier-Legendre spectral approximation with $48 \times 49$ modes
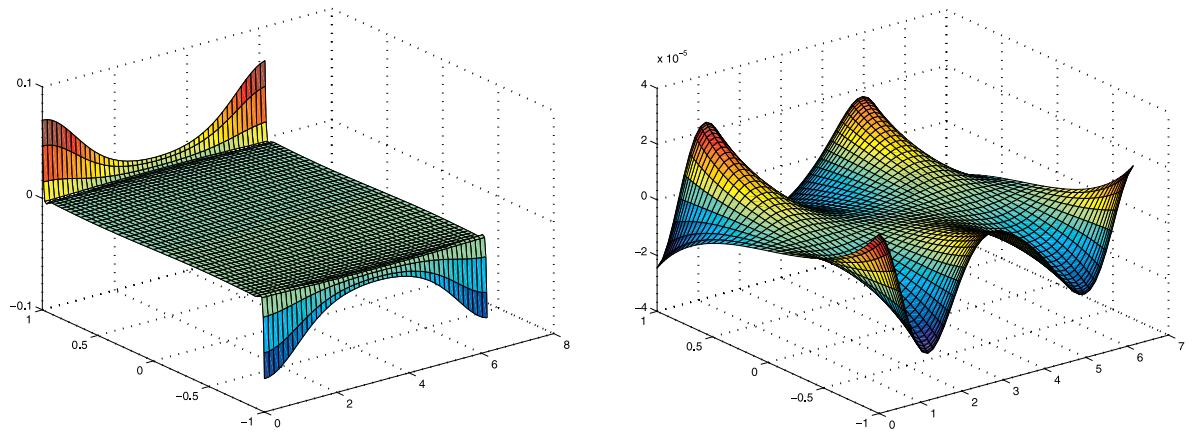

Figure 3. Error field on pressure at time $t=1$ in a channel: left, standard form; right, rotational form.
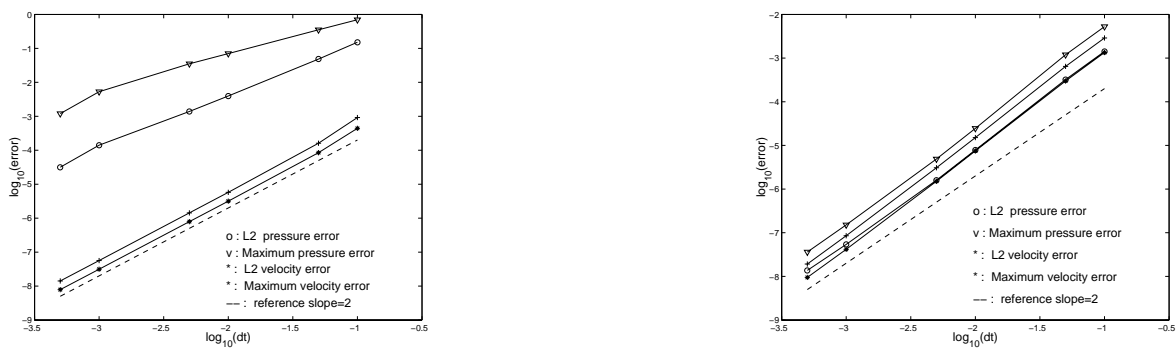

FiguRE 4. Convergence rates in a periodic channel: left, standard form; right, rotational form. 
guaranteeing that the spatial discretization errors are negligible compared with the time discretization errors.

In Figure 3 we show the pressure error field at $t=1$ for a typical time step, and in Figure 4, we plot the errors on the velocity and on the pressure as functions of $\delta t$.

We observe that the pressure error from the standard formulation still exhibits numerical boundary layers, whereas the pressure error from the rotational formulation is now smooth everywhere. Moreover, the rate of convergence on the pressure from the rotational formulation is now fully second-order.

The main difference between the problem set in the square domain and that set in the periodic channel is that the former has corner singularities while the latter does not. Thus, it can be conjectured that the large errors occurring at the corners of the square domain are due to the lack of smoothness of the domain. However, why the corner singularity affects the convergence rate for a smooth solution is still not well understood. This conclusion is confirmed by the numerical experiments using mixed finite elements reported in the next section.

5.2. Numerical results with $\mathbb{P}_{2} / \mathbb{P}_{1}$ finite elements. To further assess the respective capabilities of the standard and rotational formulations, we have also carried out convergence tests using $\mathbb{P}_{2} / \mathbb{P}_{1}$ finite elements.

To avoid using an exceedingly refined mesh to guarantee that the spatial discretization error is negligible compared with the time discretization error on a wide range of time steps, we use the analytical solution

$$
\begin{aligned}
& u=\sin (x+t) \sin (y+t), \\
& v=\cos (x+t) \cos (y+t), \\
& p=\sin (x-y+t),
\end{aligned}
$$

which is somewhat smoother than the one used to test the spectral approximation.

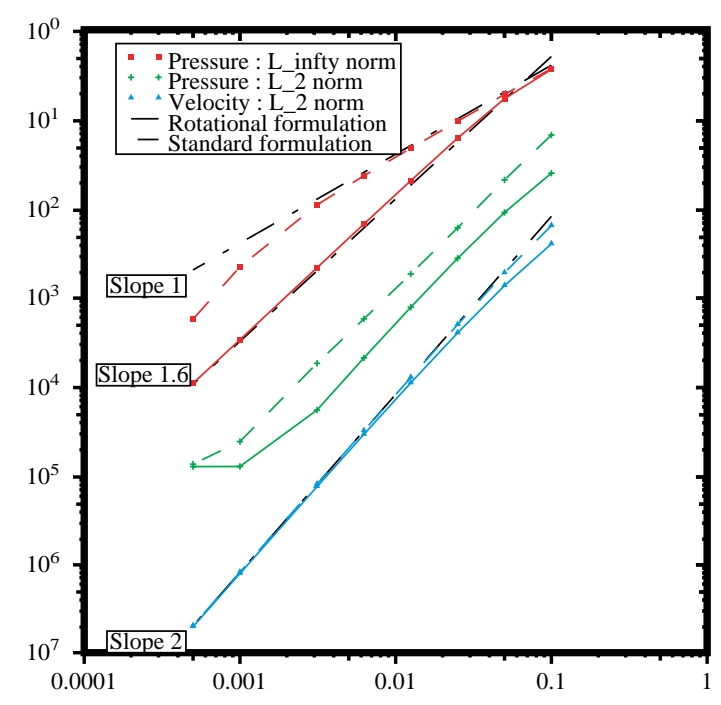

Figure 5. Convergence rates in a square: dashed line for the standard formulation; solid line for the rotational formulation. 

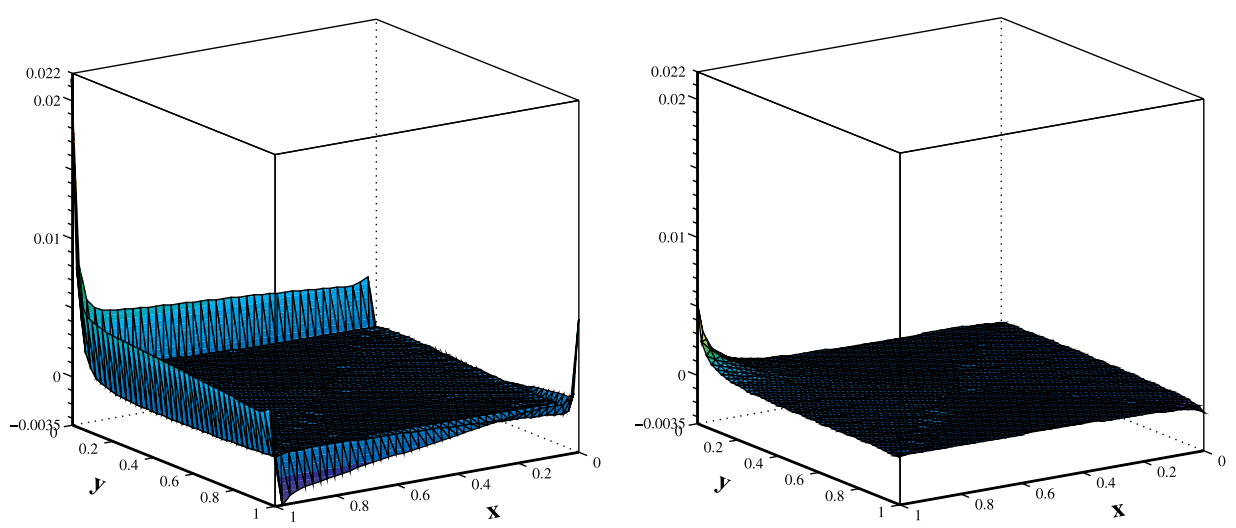

Figure 6. Error field on pressure in a rectangular domain: left, standard formulation; right, rotational formulation.

The domain considered to perform the convergence tests is the square $(0,1)^{2}$. We used a $\mathbb{P}_{2} / \mathbb{P}_{1}$ mesh composed of 14774 elements $\left(7548 \mathbb{P}_{1}\right.$ nodes and $29869 \mathbb{P}_{2}$ nodes $)$ corresponding to the average mesh size $h=1 / 80$. In Figure 5, we show the error on the velocity in the $L^{2}$-norm and that on the pressure in the $L^{2}$-norm and $L^{\infty}$-norm. The errors are measured at time $t=1$. The conclusions are essentially the same as that from the tests with the spectral approximation. The rate on the velocity is clearly second-order, and the rotational formulation does not significantly improve the accuracy on the velocity, though the error produced is systematically lower than that from the standard formulation. Concerning the pressure, the convergence rates on the errors in the $L^{2}$-norm are slightly lower than second-order for both forms of the algorithm, the rotational form systematically producing better results though. The slight saturation of the errors for very small time steps is due to the spatial interpolation error that becomes visible. For the $L^{\infty}$-norm, the convergence rates are obviously different. It is 1.6 for the rotational formulation and first-order for the standard formulation, with the departure from first-order for small time steps due to nonuniform inverse estimates as we have verified that the position of departure moves to the left when the mesh is refined.

We show in Figure [6 the error fields on the pressure at time $t=1$ for $\delta t=0.00625$. As for the spectral approximation, we note that the rotational form of the algorithm yields a pressure field that is free of a numerical boundary layer, whereas a boundary layer is clearly visible on the pressure field obtained by means of the standard algorithm. We note also that large errors are still present at the corners of the domain for both formulations.

To clarify the effect of the smoothness of the boundary on the error on the pressure, we have tested the two methods on the circular domain $\Omega=\left\{(x, y) ; \sqrt{x^{2}+y^{2}}\right.$ $\leq 0.5\}$, using the same analytical solution as before and $h=1 / 80$. We show in Figure 7 the error field on the pressure for $\delta t=0.0125$ at $t=2$. A numerical boundary layer is clearly visible on the entire boundary for the pressure calculated by means of the standard formulation, but the error is uniformly small for the pressure calculated using the rotational formulation. This test confirms that the smoothness of the boundary has a very important impact on the quality of the approximation offered by the rotational formulation. 

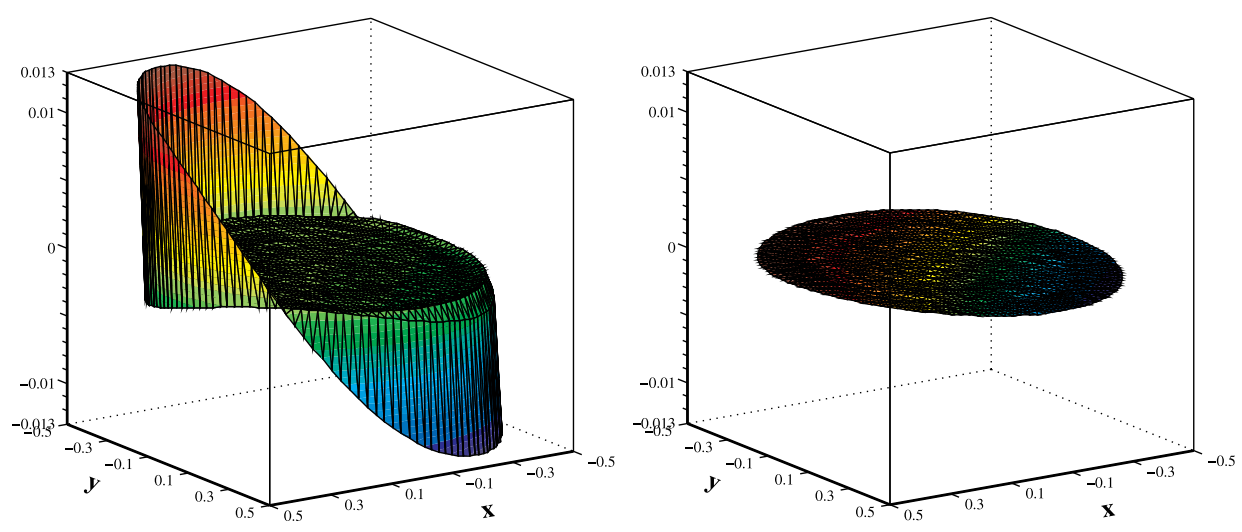

FiguRE 7. Error field on pressure in a circular domain: left, standard formulation; right, rotational formulation.

This point is made even more clear in Figure 8. In the left graph of the figure, we show the convergence rates on the velocity in the $L^{2}$-norm and that on the pressure in the $L^{2}$-norm and $L^{\infty}$-norm, with the error measured at time $t=2$. The convergence rates are all second-order for the rotational formulation, whereas this is not the case for the standard one. In the graph in the right panel of the figure, we compare the convergence rates on the pressure in the $L^{\infty}$-norm for the rotational formulation only, one series of computation being made on the square and the other on the circle. It is clear that the errors calculated on the circular domain are $\mathcal{O}\left(\delta t^{2}\right)$, whereas those calculated on the square are only $\mathcal{O}\left(\delta t^{1.6}\right)$.
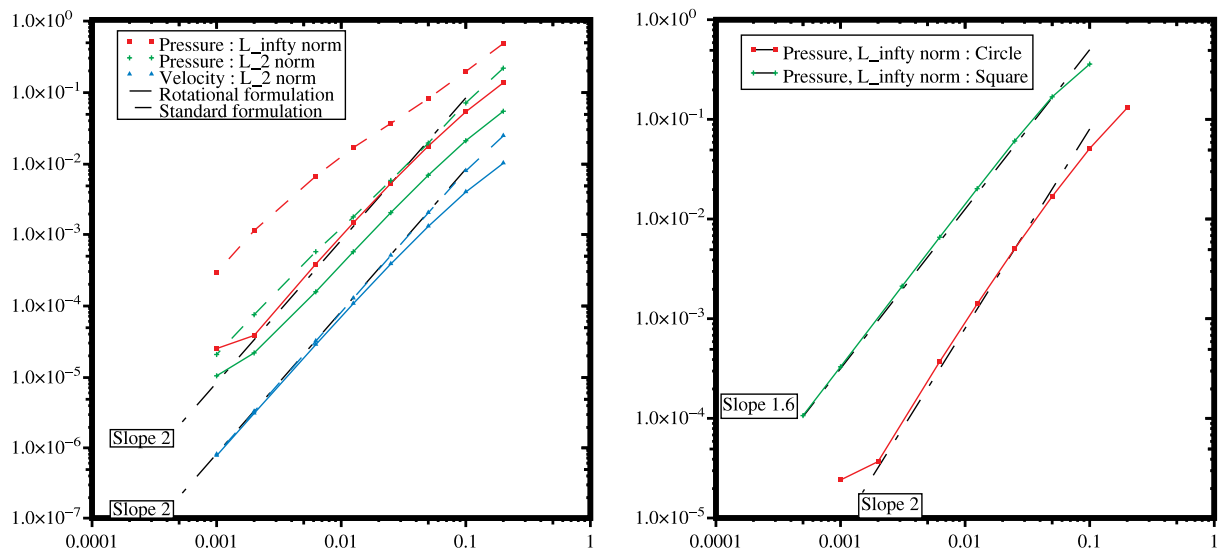

FiguRE 8. Left: convergence rates on a circular domain; dashed lines for the standard formulation; solid lines for the rotational formulation. Right: comparison of convergence rates on pressure in $L^{\infty}$-norm; solid line for the circular domain; dashed line for the square. 


\subsection{Discussions on the convergence rates of the pressure approximations.} There exists in the literature a substantial number of works dedicated to numerical and theoretical investigations on the convergence rates of the pressure using pressure-correction schemes. For the standard form, first-order error estimates on the pressure are established in [12, 15] for the semi-discrete case and in [7] for the fully discrete case. These results are valid in fairly general domains such as convex polygons. In [4, E and Liu, using asymptotic analysis in a periodic channel, obtained for the standard formulation a first-order error estimate on the pressure in the $L^{\infty}$-norm. All these results are consistent with the claim of Strikwerda and Lee in [16] that the pressure approximation in the standard formulation can be at most first-order accurate. This claim is based on a normal mode analysis in the half-plane.

In 1], using a normal mode analysis in a periodic channel, Brown, Cortez and Minion showed that the pressure approximation in the rotational formulation is second-order accurate. This is consistent with our numerical results in a periodic channel as well, but unfortunately, this result does not hold for general domains as is evidenced by our numerical results. Therefore, it seems that the convergence rate of $\frac{3}{2}$ we established here for the pressure approximation in rotational form is the best possible for general domains.

\section{REFERENCES}

1. David L. Brown, Ricardo Cortez, and Michael L. Minion. Accurate projection methods for the incompressible Navier-Stokes equations. J. Comput. Phys., 168(2):464-499, 2001. MR 2002a:76112

2. A. J. Chorin. Numerical solution of the Navier-Stokes equations. Math. Comp., 22:745-762, 1968. MR 39:3723

3. A. J. Chorin. On the convergence of discrete approximations to the Navier-Stokes equations. Math. Comp., 23:341-353, 1969. MR 39:3724

4. W. E and J.G. Liu. Projection method I: Convergence and numerical boundary layers. SIAM J. Numer. Anal., 32:1017-1057, 1995. MR 96e:65061

5. W. E and J.G. Liu. Projection method. III. Spatial discretization on the staggered grid. Math. Comp., 71(237):27-47, 2002. MR 2002k:65155

6. K. Goda. A multistep technique with implicit difference schemes for calculating two- or threedimensional cavity flows. J. Comput. Phys., 30:76-95, 1979.

7. J.-L. Guermond. Un résultat de convergence à l'ordre deux en temps pour l'approximation des équations de Navier-Stokes par une technique de projection. Modél. Math. Anal. Numér. (M2AN), 33(1):169-189, 1999. MR 2000k:65171

8. J.L. Guermond and Jie Shen. Velocity-correction projection methods for incompressible flows. To appear in SIAM J. Numer. Anal.

9. G. E. Karniadakis, M. Israeli, and S. A. Orszag. High-order splitting methods for the incompressible Navier-Stokes equations. J. Comput. Phys., 97:414-443, 1991. MR 92h:76066

10. S. A. Orszag, M. Israeli, and M. Deville. Boundary conditions for incompressible flows. J. Sci. Comput., 1:75-111, 1986.

11. R. Rannacher. On Chorin's projection method for the incompressible Navier-Stokes equations. Lecture Notes in Mathematics, vol. 1530, 1991. MR 95a:65149

12. Jie Shen. On error estimates of the projection methods for the Navier-Stokes equations: firstorder schemes. SIAM J. Numer. Anal., 29:57-77, 1992. MR 92m:35213

13. Jie Shen. On pressure stabilization method and projection method for unsteady Navier-Stokes equations. In R. Vichnevetsky, D. Knight, and G. Richter, editors, Advances in Computer Methods for Partial Differential Equations, pages 658-662, IMACS, 1992.

14. Jie Shen. Efficient spectral-Galerkin method I. direct solvers for second- and fourth-order equations by using Legendre polynomials. SIAM J. Sci. Comput., 15:1489-1505, 1994. MR 95j:65150 
15. Jie Shen. On error estimates of projection methods for the Navier-Stokes equations: secondorder schemes. Math. Comp, 65:1039-1065, July 1996. MR 96j:65091

16. J. C. Strikwerda and Y. S. Lee. The accuracy of the fractional step method. SIAM J. Numer. Anal., 37(1):37-47, 1999. MR 2000h:65127

17. R. Temam. Sur l'approximation de la solution des équations de Navier-Stokes par la méthode des pas fractionnaires ii. Arch. Rat. Mech. Anal., 33:377-385, 1969. MR 39:5968

18. R. Temam. Navier-Stokes Equations: Theory and Numerical Analysis. North-Holland, Amsterdam, 1984. MR 58:29439]

19. L. J. P. Timmermans, P. D. Minev, and F. N. Van De Vosse. An approximate projection scheme for incompressible flow using spectral elements. Int. J. Numer. Methods Fluids, 22:673-688, 1996.

20. J. van Kan. A second-order accurate pressure-correction scheme for viscous incompressible flow. SIAM J. Sci. Stat. Comput., 7:870-891, 1986. MR 87h:76008

LIMSI (CNRS-UPR 3152), BP 133, 91403, Orsay, France

E-mail address: guermond@limsi.fr

Department of Mathematics, Purdue University, West Lafayette, Indiana 47907

E-mail address: shen@math.purdue.edu 